\title{
IDENTIFICAÇÃO E CARACTERÍSTICAS DOS STAKEHOLDERS NA CONSTRUÇÃO DO PLANEJAMENTO ESTRATÉGICO DE UMA INSTITUIÇÃO PÚBLICA
}

\section{IDENTIFICATION AND CHARACTERISTICS OF STAKEHOLDERS IN THE CONSTRUCTION OF THE STRATEGIC PLANNING OF A PUBLIC INSTITUTION}

Sérgio Castro Gomes ${ }^{1}$, Laurimar De Matos Farias², Luciana Rodrigues Ferreira ${ }^{3}$, Flavio Heleno Solano Reis ${ }^{4}$

\author{
Submetido: 14/12/2020 \\ Aprovado: 11/01/2021
}

\section{RESUMO}

O estudo tem por objetivo identificar e apresentar a percepção dos stakeholders presentes nas relações internas e externas de uma Escola de Governança Pública (EGP) e que fizeram parte do processo de construção do Planejamento Estratégico Institucional (PEI) da EGP. Trata-se de uma pesquisa empírica ancorada na efetiva participação de todos os stakeholders, em que barreiras, ameaças, oportunidades e desafios foram analisados. O objeto de pesquisa foi uma Escola de Governança Pública (EGP) de um estado da Federação, que é responsável pela promoção do desenvolvimento e valorização do servidor público. A pesquisa empregou as abordagens qualitativas e quantitativas, de forma complementar, e múltiplos métodos de pesquisa foram utilizados como entrevista em profundidade, grupo focal e pesquisa tipo survey, de forma a capturar a percepção dos diferentes stakeholders. Os resultados da pesquisa mostram que os stakeholders primários da EGP são os prestadores de serviços de segurança, higienização e Tecnologia da Informação (TI); Secretários, Diretores e Presidentes de organizações públicas; alunos; professores e egressos; para superar as barreiras internas é preciso maior alinhamento da EGP com os prestadores de serviços de TI e o reconhecimento da missão da EGP pelas demais instituições públicas estaduais.

PALAVRAS-CHAVE: Planejamento Estratégico Institucional. Stakeholders. Setor Público.

\begin{abstract}
This research aims to identify and present the perception of stakeholders present in the internal and external relations of a School of Public Governance (SPG) and who were part of the construction process of the Institutional Strategic Planning (ISP) of SPG. It is an empirical research based on the effective participation of all stakeholders, in which barriers, threats, opportunities and challenges were analyzed. The research object was a School of Public Governance (SPG) of a state of the Federation, which is responsible for promoting the development and enhancement of the civil servant. The research used qualitative and quantitative approaches, in a complementary way, and multiple research methods were used as an in-depth interview, focus group and survey type survey, in order to capture the perception of different stakeholders. The survey results show that SPG's primary stakeholders are providers of security, hygiene and Information Technology (IT) services; Secretaries,
\end{abstract}

\footnotetext{
1 Doutor em Economia Aplicada pela Universidade Federal de Viçosa. Professor permanente do Programa de Pós-Graduação em Administração na Universidade da Amazônia (PPAD/UNAMA). Pesquisador do Centro de Estudos, Pesquisas e Projetos Estratégicos em Governança Pública da Escola de Governança Pública do Estado do Pará (CEPPE/EGPA).

2 Doutor em Educação pela Universidade Federal do Pará. Pesquisador do Centro de Estudos, Pesquisas e Projetos Estratégicos em Governança Pública da Escola de Governança Pública do Estado do Pará (CEPPE/EGPA). Professor Classe IV da Secretaria de Estado de Educação do Pará (SEDUC) e da Secretaria Municipal de Educação de Belém (SEMEC).

3 Doutora em Educação pela Universidade Federal de São Carlos (UFSCar). Professora permanente do Programa de Pós-Graduação em Administração na Universidade da Amazônia (PPAD/UNAMA). Coordenadora do Mestrado Profissional em Gestão de Conhecimentos para o Desenvolvimento socioambiental (PPGC/UNAMA). Pesquisadora do Centro de Estudos, Pesquisas e Projetos Estratégicos em Governança Pública da Escola de Governança Pública do Estado do Pará (CEPPE/EGPA).

4 Mestre em Gestão e Desenvolvimento Regional pela Universidade de Taubaté (UNITAU). Técnico Nível Superior lotado no Centro de Estudos, Pesquisas e Projetos Estratégicos em Governança Pública da Escola de Governança Pública do Estado do Pará (CEPPE/EGPA), Docente nas faculdades ESMAC e Cosmopolita.
} 


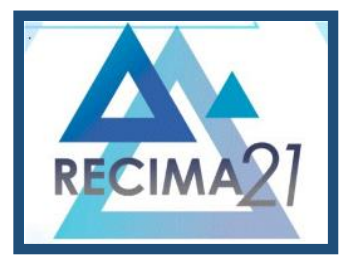

\section{RECIMA21 - REVISTA CIENTÍFICA MULTIDISCIPLINAR}

IDENTIFICAÇÃO E CARACTERÍSTICAS DOS STAKEHOLDERS NA CONSTRUÇÃO DO PLANEJAMENTO

ESTRATÉGICO DE UMA INSTITUIČ̃̃O PÚBLICA

Sérgio Castro Gomes, Laurimar De Matos Farias, Luciana Rodrigues Ferreira, Flavio Heleno Solano Reis

Directors and Presidents of public organizations; students; teachers and graduates; to overcome internal barriers, greater alignment of SPG with IT service providers and recognition of SPG's mission by other state public institutions is required.

KEYWORDS: Institutional Strategic Planning. Stakeholders. Public Sector.

\section{INTRODUÇÃO}

O planejamento estratégico empregado pela empresa privada refere-se a um conjunto de ações que a empresa deve realizar para criar valor ao produto final, e o resultado final é o alcance de um objetivo definido previamente Brandenburguer; Stuart (1996). Nas organizações públicas a ideia de criar valor, segundo Bryson (1988), tem o sentido de entregar serviços aos cidadãos e satisfazer as suas necessidades básicas de saúde, segurança, educação e outras demandas que, quando ofertadas na quantidade adequada, ao preço justo e na qualidade exigida, resulta na elevação do bem-estar da sociedade.

No entanto, o maior desafio dos gestores de serviços públicos é planejar e executar de forma eficaz, eficiente e efetiva as políticas públicas de forma a melhorar a vida dos cidadãos. Neste sentido, os manuais tradicionais de gestão estratégica oferecem as ferramentas necessárias para que a empresa privada realize seu planejamento estratégico, defina sua missão, enuncie a sua visão e diga onde deseja se posicionar no mercado em um tempo futuro. No setor público esse processo é mais complexo diante de um conjunto de grupos sociais que disputam como alocar os limitados recursos públicos para atender as ilimitadas demandas dos cidadãos por mais serviços públicos além da disponibilidade e qualidade de capital humano, tecnológico e físico dos órgãos públicos (Biderman; Arvate, 2004).

Neste sentido, a sustentabilidade das organizações produtivas contemporâneas, incluindose as organizações públicas e a execução de suas ações estratégicas, atrela-se, dentre outros fatores, a questão das competências do seu corpo de funcionários no exercício das variadas atividades funcionais exigidas ao bom desempenho organizacional, que como consequência, originará a competência organizacional (Quin, 2003). Com efeito, a de se esperar que os integrantes das organizações públicas devam possuir competências individuais e coletivas, quanto à compreensão da integração organizacional com o seu meio ambiente de entorno (ambiental, social, político e econômico), de forma que essas competências sejam utilizadas na formulação, adoção, monitoramento e avaliação de políticas públicas capazes de transformar, para melhor, a condição de vida das famílias.

O presente estudo tem como objeto de pesquisa uma Escola de Governança Pública (EGP) de um estado da Federação, que é responsável pela promoção do desenvolvimento e valorização do servidor público a partir da formação de pessoas e da produção de conhecimento para o fortalecimento das competências e capacitações necessárias a execução de sua missão. 


\section{RECIMA21 - REVISTA CIENTÍFICA MULTIDISCIPLINAR}

IDENTIFICAÇÃO E CARACTERÍSTICAS DOS STAKEHOLDERS NA CONSTRUÇÃO DO PLANEJAMENTO

ESTRATÉGICO DE UMA INSTITUIČ̃̃O PÚBLICA

Sérgio Castro Gomes, Laurimar De Matos Farias, Luciana Rodrigues Ferreira, Flavio Heleno Solano Reis

A EGP em discussão tem um conjunto de relacionamentos, como toda organização, tem um conjunto de colaboradores internos e externos, que são essenciais no processo elaboração do Planejamento Estratégico Institucional (PEI).

$\mathrm{Na}$ visão de Freeman (1984) e Bourne e Walker (2005), esses colaboradores são denominados de stakeholders, e a forma como eles se comportam no relacionamento com a organização tem relação com o desempenho, bem como são também afetados pelas decisões da organização. Nesse contexto, torna-se importante identificar esses grupos e criar formas de relacionamento e de reconfiguração dessas relações conforme o ambiente interno e externo da organização.

Como forma de contemplar essa visão colaborativa e participativa dos stakeholders, no caso de uma organização pública, foi empregado o modelo de planejamento estratégico proposto por Bryson (1988) para instituições públicas e sem fins lucrativos, de forma a considerar que os fatores do ambiente organizacional e suas relações com os stakeholders, orientarão a formulação das estratégias e seu alcance, diferente da proposição do Balanced Scorecard, em sua versão original, que está orientada a partir da percepção e vivência dos gerentes (Kaplan \& Norton, 1997).

Neste sentido, o estudo tem por objetivo identificar e caracterizar os stakeholders presentes nas relações internas e externas de uma EGP. Esses objetivos foram alcançados a partir do processo de construção do PEI da EGP. Trata-se de uma pesquisa empírica ancorada na efetiva participação de todos os stakeholders, em que barreiras, ameaças, oportunidades e desafios foram analisadas. O estudo considera a complementaridade existente o método qualitativo e o quantitativo, e a utilização de múltiplos métodos de pesquisa como entrevista em profundidade, grupo focal e pesquisa tipo survey, como forma de melhor capturar a percepção dos diferentes grupos representados na pesquisa.

Este artigo está estruturado em sete sessões contando com esta introdução e conclusão. A trajetória de criação até os dias atuais da EGP é apresentada na sessão dois. O conceito de planejamento estratégico e os stakeholders a sua aplicação na gestão pública são discutidos na sessão três. Na sessão quatro, são estudados os procedimentos metodológicos apresentados e é justificada a necessidade de se utilizar uma abordagem multimétodos. A sistematização dos resultados das pesquisas foi inserida na sessão cinco considerando cada uma das quatro pesquisas realizadas. Na última sessão foram discutidos os resultados das pesquisas de forma integrada e reflexiva e por último o encerramento na sessão sete com a conclusão.

\section{CONTEXTO HISTÓRICO DA ESCOLA DE GOVERNANÇA PÚBLICA}

No serviço público brasileiro, o papel de atuação na formação continuada e de aprendizagens corporativas está estreitamente ligado a dois processos históricos de atuação do Estado: a Reforma do Aparelho do Estado de 1995, que deu o tom de uma agenda gerencial pautada 


\title{
RECIMA21 - REVISTA CIENTÍFICA MULTIDISCIPLINAR
}

IDENTIFICAÇÃO E CARACTERÍSTICAS DOS STAKEHOLDERS NA CONSTRUÇÃO DO PLANEJAMENTO

ESTRATÉGICO DE UMA INSTITUIČ̃̃O PÚBLICA

Sérgio Castro Gomes, Laurimar De Matos Farias, Luciana Rodrigues Ferreira, Flavio Heleno Solano Reis

na eficiência institucional e que abrigou todo o processo de reorganização do labor público; e, a nova abordagem de gestão estatal, de meados dos anos 2000, com agenda das Escolas de Governança Pública dos Estados.

Neste contexto, orienta-se para um processo de produção da sociedade dirigida para o setor produtivo, cujo valor está em diluir as diferenças, no âmbito do Estado, entre o público e o privado. Analisa-se, com isso que, em consonância com as determinações e interesses dos organismos internacionais, o Estado passa a exercer um papel de regulador social, necessário para o controle de procedimentos e resultados, com o caráter intermediário entre o mercado e os setores da administração pública (Nogueira, 2005).

Pode-se afirmar que, com o objetivo de formação de servidores mais estratégico para atender as demandas de uma Reforma Gerencial, a EC 19/1998, adotou ferramentas administrativas voltadas à efetividade e eficiência, e, introduziu no ordenamento jurídico brasileiro as Escolas de Governo, de observância obrigatória pela União, Estados e Distrito Federal, que assim dispõe em seu Artigo 39, parágrafo 20:

\begin{abstract}
A União, os Estados e o Distrito Federal manterão escolas de governo para a formação e o aperfeiçoamento dos servidores públicos, constituindo-se a participação nos cursos um dos requisitos para a promoção na carreira, facultada, para isso, a celebração de convênios ou contratos entre os entes federados. (BRASIL, 1998, p. 13, grifos nossos).
\end{abstract}

Tal feito tomou uma proporção singular de expansão de instituições governamentais direcionadas às aprendizagens corporativas e acadêmicas de formação para o trabalho. Com a justificativa sobre as necessárias mudanças da administração pública burocrática para a administração gerencial as Escolas de Governo passam a atuar em todas as regiões do Brasil.

Aires et al (2014), em estudo exploratório sobre "Escolas de governo: o panorama brasileiro", apresentam um levantamento das atividades declaradas das escolas de governo do Brasil, obtidas nos websites destas instituições, por região geográfica. Compilaram cerca de trinta escolas, com maior concentração em números no Nordeste, mas com escolas de maior porte de tamanho e recursos no Distrito Federal (Centro-Oeste), na região sudeste e sul. Os autores consideram que:

[...] as escolas de governo atuam como impulsionadoras de uma administração ágil e voltada para o cidadão ou para finalidades e missões estrategicamente definidas para suas instituições, é importante que elas se posicionem como as escolas corporativas de gestão dos governos, se antecipando em busca de novas tendências e melhores práticas, exercendo um papel de relevância e influência na própria formulação das políticas voltadas para melhoria da gestão, atuando na adaptação dessas ferramentas ao contexto do setor público, além de possuírem valores e indicadores de desempenho próprios (AIRES et al. 2014, p. 1010, grifo nosso). 


\section{RECIMA21 - REVISTA CIENTÍFICA MULTIDISCIPLINAR}

IDENTIFICAÇÃO E CARACTERÍSTICAS DOS STAKEHOLDERS NA CONSTRUÇÃO DO PLANEJAMENTO ESTRATÉGICO DE UMA INSTITUIČ̃̃O PÚBLICA Sérgio Castro Gomes, Laurimar De Matos Farias, Luciana Rodrigues Ferreira, Flavio Heleno Solano Reis

De tal forma, isso demonstra o papel protagonista das Escolas de Governos na gestão pública. Pacheco $(2000$; 2002) corrobora com a visão que estas instituições devem prospectar conhecimento e tecnologias gerenciais em sua melhor performance, muitas destas, previamente desenvolvidas no setor privado, pelo próprio contexto de gestão gerencial que as escolas foram criadas, mas que tenham a capacidade de contextualizar este know-how às especificidades das organizações públicas.

\section{A IMPORTÂNCIA DOS STAKEHOLDERS NO PLANEJAMENTO ESTRATÉGICO DO SETOR PÚBLICO}

Nesta pesquisa assume-se o conceito de stakeholders enunciado por Bryson (2004) que considera ser o conjunto formado por todos os sujeitos - pessoas, grupos ou organizações, que devem ser considerados pelos secretários e outros líderes, além dos próprios servidores públicos que atuam no atendimento ao cidadão, de forma direta ou indireta.

Na perspectiva de Freeman (1984), os stakeholders são definidos como qualquer grupo ou indivíduo que seja afetado ou possa afetar a realização dos objetivos de uma organização. Assim, a organização estabelece relacionamentos institucionais formais com todos colaboradores que se interrelacionam com a organização para que ela atinja seus objetivos e cumpra a sua missão. Para Phillips (2003) esse grupo é formado por funcionários, gerentes, fornecedores, clientes e proprietários, que são definidos como stakeholders primários ou legítimos, que interferem e/ou são interferidos na execução de suas atividades. O grupo formado pelos colaboradores secundários contempla comunidades, grupos específicos de interesse indireto e a mídia que são influenciados ou influenciam no desenvolvimento das atividades da organização (Savage et al., 1991).

A preocupação em identificar e caracterizar os stakeholders, no caso das instituições públicas deve-se, em parte, pela melhoria da qualidade dos serviços oferecidos ao público/cidadão com menos aporte de tempo e melhor uso do orçamento estatal, isto é, faz-se necessário gerar "valor para a sociedade e formas de garantir o desenvolvimento sustentável, sem perder de vista a obrigação de utilizar os recursos em um formato eficiente", Batista (2012, p. 16).

O aprimoramento dessa relação organização-stakeholders é de fundamental importância considerando o fato de a organização ser uma empresa pública que carrega um conjunto de características próprias desse tipo de instituição, que se diferencia pelo fato do valor criado estar ligado ao bem-estar da população, ou seja, o interesse nesse campo teórico recai sobre a sinergia criada no ambiente em que a gestão pública seja capaz de entregar o melhor serviço a um preço justo.

Neste sentido a compreensão de planejamento estratégico, segundo Bryson e Roerin (1988, p. 995) "pode ser definido como um esforço disciplinado para produzir decisões e ações fundamentais que definam o que uma organização (ou outra entidade) é, o que faz e por que faz 


\section{RECIMA21 - REVISTA CIENTÍFICA MULTIDISCIPLINAR}

IDENTIFICAÇÃO E CARACTERÍSTICAS DOS STAKEHOLDERS NA CONSTRUÇÃO DO PLANEJAMENTO

ESTRATÉGICO DE UMA INSTITUIČ̃̃O PÚBLICA

Sérgio Castro Gomes, Laurimar De Matos Farias, Luciana Rodrigues Ferreira, Flavio Heleno Solano Reis

isso". Ao considerar essas prerrogativas, é salutar para a gestão pública ter definido qual sua missão no processo ou fluxo de atendimento ao cidadão nas suas mais basilares necessidades e, ainda, determinar, a partir dos interesses públicos, qual sua visão de futuro e, por fim, a definição dos seus objetivos estratégicos institucionais.

O planejamento estratégico com estas peculiaridades no setor público, diferente do setor privado que visa maximizar a eficácia em toda a empresa ou subunidade em termos de lucro, participação de mercado e outros resultados relacionados aos negócios, estão alinhados ao alcance de metas por meio do desempenho e esforço do estado e seus agentes públicos na melhoria dos serviços à comunidade Brayson et al (2018).

Neste aspecto, a gestão gerencial se adéqua aos princípios do setor público, não na geração de um valor financeiro ou produto e sua mais valia, mas na presença do estado com promoção e oferta de serviços qualificados que chegue ao cidadão. A promoção destes serviços deve gerar modificações socioeconômicas para o bem-estar da sociedade como um todo, na oferta de políticas públicas que evidenciem os pilares da gestão pública responsável, quais sejam: eficácia, eficiência e efetividade, que segundo Nogueira (2005), podem ser entendidos como recursos de análise distintos para o acompanhamento e avaliação futura.

\section{METODOLOGIA}

A pesquisa é de natureza exploratória, e a construção metodológica combinou a abordagem qualitativa e quantitativa de forma complementar, esse arranjo metodológico é conhecido na literatura como método misto Creswell (2010) por possibilitar a captura das percepções dos sujeitos da pesquisa de maneira à melhor qualificar a informação produzida a partir da aplicação de métodos quantitativos que resultam da sistematização dos dados e da identificação dos padrões de ocorrência das principais variáveis.

Os principais stakeholders participantes da pesquisa foram: os servidores públicos efetivos ou não; os colaboradores externos da EGP como prestadores de serviços de segurança, higienização e Tecnologia da Informação (TI); Secretários, Diretores e Presidentes de organizações públicas; alunos; professores e egresso.

O método de coleta de dados junto aos stakeholders conjugou a utilização de múltiplas técnicas qualitativas e quantitativas de coleta, tratamento de dados e análise de resultados. Nesse contexto foi aplicada a técnica de entrevista em profundidade cujos sujeitos foram dois secretários de estado vinculados as ações de planejamento, orçamento e finanças, e de ação social, a Direção Geral e as diretorias de execução e operacionalização da EGP, essas entrevistas duraram entre $30 \mathrm{e}$ 50 minutos e foram orientadas por um conjunto de perguntas não estruturadas que visou capturar a percepção desses sujeitos sobre o momento atual da EGP, a necessidade de ajustes diante de um conjunto de expectativas futuras e quais seriam esses ajustes. 


\section{RECIMA21 - REVISTA CIENTÍFICA MULTIDISCIPLINAR}

IDENTIFICAÇÃO E CARACTERÍSTICAS DOS STAKEHOLDERS NA CONSTRUÇÃO DO PLANEJAMENTO
ESTRATÉGICO DE UMA INSTITUIÇÃO PÚBLICA
Sérgio Castro Gomes, Laurimar De Matos Farias, Luciana Rodrigues Ferreira, Flavio Heleno Solano Reis

Os servidores públicos da EGP participaram do processo de diagnóstico situacional da gestão da escola a partir da formação de grupos focais por diretoria em que foram identificados os pontos fortes e fracos e as ameaças e oportunidades para EGP conforme o modelo da formulação das estratégias pela matriz SWOT, além de pontuarem as barreiras existentes para o melhor desenvolvimento de suas atividades, o que foi realizado em uma primeira rodada de seminário interno na EGP.

Os colaboradores externos fizeram parte da etapa de identificação das barreiras existentes, utilizando-se de um grupo focal formado pelas prestadoras de serviços à EGP, em que as entrevistas duraram entre 30 e 40 minutos.

A percepção dos alunos, egressos e professores sobre os aspectos relacionados à matriz SWOT foi capturado a partir da aplicação de uma pesquisa tipo survey junto a uma amostra de 110 participantes, distribuídos entre Alunos, Egressos e Professores dos cursos da EGP. A cada grupo foi aplicado um questionário com perguntas abertas para caracterizar o respondente, e fechadas para que o entrevistado identificasse se um determinado item era um ponto forte (SIM) ou fraco (NÃO) da EGP, e depois os qualifica como baixo, médio ou alto.

O QUADRO 1 resume essa distribuição de sujeitos investigados, tipo e classificação das pesquisas realizadas cujos resultados orientaram o planejamento estratégico da EGP para o período de 2020 a 2023.

Quadro 1 - Distribuição dos stakeholders da pesquisa, 2020.

\begin{tabular}{|c|c|c|c|c|}
\hline Grupo & $\begin{array}{c}\text { Classificação da } \\
\text { Pesquisa }\end{array}$ & $\begin{array}{c}\text { Tipo de } \\
\text { pesquisa }\end{array}$ & $\begin{array}{c}\text { Número de } \\
\text { participantes }\end{array}$ & Duração da pesquisa \\
\hline $\begin{array}{c}\text { Secretários de Estado e Diretores } \\
\text { da EGP }\end{array}$ & Qualitativa & $\begin{array}{c}\text { Entrevista em } \\
\text { Profundidade }\end{array}$ & 6 & $\begin{array}{c}30 \text { a } 50 \\
\text { min/entrevistado }\end{array}$ \\
\hline Servidores públicos da EGP & Qualitativa & Grupo focal & 24 & $\begin{array}{c}30 \text { a } 40 \\
\text { min/entrevistado }\end{array}$ \\
\hline $\begin{array}{c}\text { Colaboradores externos - } \\
\text { Prestadores de Serviços à EGP }\end{array}$ & Qualitativa & Grupo focal & 6 & $\begin{array}{c}30 \text { a } 40 \\
\text { min/entrevistado }\end{array}$ \\
\hline $\begin{array}{c}\text { Alunos, Egressos e Professores da } \\
\text { EGP }\end{array}$ & Quantitativa & Survey & 110 & 30 dias \\
\hline
\end{tabular}

Fonte: Resultados da pesquisa.

A pesquisa tipo survey foi viabilizada utilizando o recurso do Google Forms disponível no site do Google, com ele foram criados os questionários e o endereço eletrônico foi enviado para uma amostra de alunos, egressos e professores num total de 1200 pessoas e o retorno foi de 110 questionários com uma taxa de retorno de 9,2\%. A distribuição entre os grupos foi de 44 alunos, 36 egressos e 30 professores. Os dados da pesquisa foram sistematizados utilizando-se de medidas estatísticas descritivas. A pesquisa survey ocorreu no período entre os meses de outubro e novembro de 2018.

\section{RESULTADOS DAS PESQUISAS}




\section{RECIMA21 - REVISTA CIENTÍFICA MULTIDISCIPLINAR}

IDENTIFICAÇÃO E CARACTERÍSTICAS DOS STAKEHOLDERS NA CONSTRUÇÃO DO PLANEJAMENTO

ESTRATÉGICO DE UMA INSTITUIČ̃̃O PÚBLICA

Sérgio Castro Gomes, Laurimar De Matos Farias, Luciana Rodrigues Ferreira, Flavio Heleno Solano Reis

A apresentação dos resultados da pesquisa seguirá a sequência resumida no Quadro1, em que os pontos relevantes das entrevistas são listados e as convergências e divergências identificadas conforme o grupo respondente.

\subsection{Secretários de Estado e Diretores da EGP}

No grupo de secretários, o aspecto relevante e considerado na construção do PEI foi a ênfase dado a importância da EGP e o papel destacado que ela pode vir a ter se focar em aspectos relacionados a capacitação dos servidores públicos e na formação de lideranças (gerentes) para fazer a gestão nos níveis estratégico, tático e operacional. No entanto, para que isso se torne realidade há a necessidade de se estruturar a infraestrutura e a capacitação dos servidores da EGP. Conforme um dos secretários, "[...] a EGP precisa inovar seus procedimentos operacionais no segmento da Tecnologia da Informação (TI) de forma a elevar a capilaridade de atendimento espacial das demandas das regiões mais distantes da capital e ofertar modelos inovadores de cursos [...]" (2019, p.10).

O sentimento de que no PEI deve-se dar ênfase aos recursos humanos e tecnológicos é consensual entre os Diretores da EGP, no entanto, acrescenta-se a urgência de recursos financeiros para realizar as melhoras na infraestrutura e a aquisição de livros e material didático para que a escola tenha condições de executar as ações planejadas.

Outro aspecto apontado por um dos secretários foi o fato da EGP ter uma oferta de cursos focada em atividades de curta duração e poucos cursos de aperfeiçoamento ou de especialização com ênfase na gestão pública e governança. Segundo o entrevistado,

[...] a EGP precisa identificar a demanda dos órgãos por formação superior em nível de Lato e Stricto Sensu, principalmente, na formação de coordenadores e diretores com competências para desenvolverem a gestão de pessoas, organizacional, logística, ambiental e todas as operações necessários ao bom desenvolvimento das atividades (2019. p. 4)

Em síntese, a maioria das falas dos entrevistados convergem no sentido de que a EGP precisa se estruturar para atender as demandas futuras da gestão pública estadual, e para tal seu plano estratégico deve contemplar processos inovativos de gestão das operações vinculadas a estrutura organizacional, aos aspectos pedagógicos, do uso das novas tecnologias educacionais, e na formulação e implantação de uma política institucional da Educação a Distância.

\subsection{Servidores públicos da EGP e colaboradores externos}

Os encontros com os servidores da EGP, que aconteceram entre janeiro e maio de 2019, foram proveitosos uma vez que a equipe de facilitadores do PEI conseguiu convencer os servidores da necessidade de se entender a atual situação da EGP e definir o posicionamento da escola no futuro. Para tal foi utilizada a matriz SWOT como ferramenta estratégica para se identificar as forças 


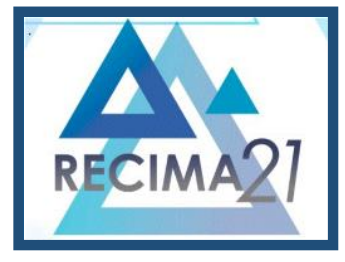

\section{RECIMA21 - REVISTA CIENTÍFICA MULTIDISCIPLINAR}

IDENTIFICAÇÃO E CARACTERÍSTICAS DOS STAKEHOLDERS NA CONSTRUÇÃO DO PLANEJAMENTO ESTRATÉGICO DE UMA INSTITUIČ̃̃O PÚBLICA Sérgio Castro Gomes, Laurimar De Matos Farias, Luciana Rodrigues Ferreira, Flavio Heleno Solano Reis

e fraquezas no ambiente interno e que são controladas pela EGP, e as oportunidades e ameaças no ambiente externo, o qual a EGP não tem poder de intervir.

Os itens que compõem a matriz SWOT, foram relacionados ao ambiente interno (Forças e Fraquezas) e do ambiente externo (Oportunidades e Ameaças), a utilização desses itens segue a orientação de que os pontos fortes devem ser potencializados e as fraquezas mitigadas para que a organização possa se tornar mais competitiva para aproveitar as oportunidades.

Neste sentido, identificou-se como oportunidades para que a EGP concretize seus objetivos estratégicos as seguintes situações: ampliar a realização de convênios e parcerias de maneira a captar recursos externos de outros órgãos da administração pública; fazer parte de redes estaduais e nacionais de escolas de governo como forma de ampliar o acesso a novas práticas de gestão, uso de novas tecnologias e outros processos que conduzam a aumento da produtividade e qualidade das ações da EGP; potencializar o uso da modalidade de ensino a distância (EaD) de forma a atender as demandas de regiões distantes da região metropolitana onde está localizada a EGP e qualificar os servidores públicos que prestam serviços a população dessas regiões.

Em termos de fatores que ameaçam a realização do planejamento estratégico os principais são relativos as condições econômicas locais, nacionais e internacionais que interferem no nível da arrecadação do estado, uma vez que a maior parcela da receita resulta de atividades econômicas extrativas minerais e de produtos agropecuários que tem seus preços definidos em bolsas de valores fora do país. A ausência de ações de desenvolvimento e competências dos servidores públicos estaduais definidas no planejamento estratégicos dos órgãos é outro fator de ameaça que pode comprometer as atividades da EGP, o que leva à redução da demanda pelos cursos e outras ações promovidas.

O conjunto de fatores positivos ou forças que devem ser utilizadas no arranjo das ações de planejamento estratégico da EGP para aproveitar as oportunidades do ambiente externo mostram que o capital humano envolvido nas ações finalísticas de realização de cursos tem elevada qualidade e é formado por profissionais competentes que dominam as competências necessárias para o desenvolvimento das atividades. O fato de a EGP ter elevada credibilidade junto aos servidores públicos a credencia para ofertar novos cursos presenciais e expandir a oferta de cursos na modalidade à distância.

No entanto, para que as ações do planejamento estratégico sejam bem sucedidas e as oportunidades sejam aproveitadas é preciso mitigar algumas situações apresentadas pelos servidores relativas à melhoria da estrutura física, implantação de processos de gestão das operações administrativas e logísticas da EGP; necessidade urgente de avaliar a satisfação do egresso dos cursos da EGP como forma de adotar processos avaliativos contínuos; e um dos fatores centrais é a precariedade das ações de ensino a distância decorrente da infraestrutura inadequada para melhor utilizar as vantagens da oferta de ensino a distância. 


\section{RECIMA21 - REVISTA CIENTÍFICA MULTIDISCIPLINAR}

IDENTIFICAÇÃO E CARACTERÍSTICAS DOS STAKEHOLDERS NA CONSTRUÇÃO DO PLANEJAMENTO

ESTRATÉGICO DE UMA INSTITUIČ̃̃O PÚBLICA Sérgio Castro Gomes, Laurimar De Matos Farias, Luciana Rodrigues Ferreira, Flavio Heleno Solano Reis

A participação deste público servidor permitiu um olhar do todo da instituição, que foi de grande valia para se identificar as lacunas da gestão institucional. Chama-se atenção para a classificação das barreiras identificadas pelos servidores e pelos agentes terceirizados que atuam dentro da instituição. Estas barreiras foram ordenadas segundo o ambiente em que se encontravam, seja interna a instituição, que chamamos de microambiente, seja em uma relação a outros órgãos e secretarias de dentro do sistema do próprio estado, que nominamos de mesoambiente ou nas relações com as instituições parceiras e/ou terceirizadas contratadas pela EGP que são os macrosambientes, a seguir, apresenta-se a sistematização que permite uma visão mais holística destas barreiras institucional.

Foram identificadas 153 barreiras no total, destas 102 estão localizadas no microambiente, isto é, $67 \%$ das barreiras estão dentro da própria instituição e estão relacionadas a questão de fluxo de processos, a falta pessoal para melhor desenvolver as atividades até a falta de comunicação entre os setores e seus agentes. Constata-se que 43 barreiras de 54, estão classificadas como de cunho organizacional e 26 barreiras na dimensão infraestrutura. Pode-se inferir que é preciso organizar o fluxo da gestão dentro da EGP para dirimir as muitas questões que se apresentam internamente e que resolvidas, deve contribuir, significativamente, para a eficácia dos serviços oferecidos por esta instituição.

O atendimento a solução das barreiras se apresenta como uma urgência a EGP na efetivação da modernização institucional, pois esta é um dos eixos presentes nas diretrizes do plano do atual governo do estado, que dentre outras proposições do plano estão a inovação tecnológica e a modernização institucional, onde a EGP apresenta-se como uma escola estratégica na atuação como escola de Inovação e políticas públicas do estado.

\subsection{Alunos, Egressos e Professores da EGP}

\subsubsection{Alunos e Egressos}

A amostra de alunos e egressos participantes da pesquisa tipo survey realizada com o objetivo de identificar padrões de características dos entrevistados e a distribuição de itens relativos aos pontos fortes e fracos e as ameaças e oportunidades na percepção desses indivíduos. $O$ diferencial desse modelo de análise reside no fato da matriz SWOT tradicional expressa a percepção dos funcionários, enquanto que, neste estudo buscou-se capturar a percepção de um grupo de stakeholders que não fazem parte do ambiente interno da EGP, a não ser quando frequentam as aulas presenciais dos diferentes cursos. Um total de 80 colaboradores foi pesquisados, 44 alunos e 36 egressos, e as seguintes características foram observadas: $61,0 \%$ dos entrevistados são mulheres; a menor idade foi de 20 anos e a maior de 66 anos; a idade dos entrevistados se distribui normalmente com média de 40 anos e desvio padrão de 9,8 anos. Os resultados do teste de 


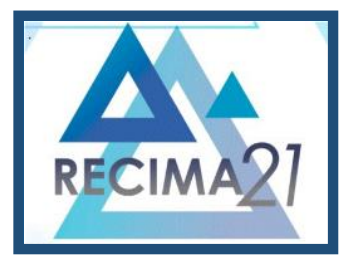

\section{RECIMA21 - REVISTA CIENTÍFICA MULTIDISCIPLINAR}

IDENTIFICAÇÃO E CARACTERÍSTICAS DOS STAKEHOLDERS NA CONSTRUÇÃO DO PLANEJAMENTO

ESTRATÉGICO DE UMA INSTITUIČ̃̃O PÚBLICA

Sérgio Castro Gomes, Laurimar De Matos Farias, Luciana Rodrigues Ferreira, Flavio Heleno Solano Reis

Kolmogorov-Smirnov mostram ser significativa a suposição de que a idade apresenta distribuição normal; $55,0 \%$ deles são funcionários públicos estaduais e $41,0 \%$ municipais; $86,0 \%$ deles realizaram curso de qualificação (24 a 40 horas) e os demais cursos técnicos profissionalizantes e especialização; $65,0 \%$ dos entrevistados cursaram o ensino básico em escolas públicas e 21,0\% em escolas privadas.

Os resultados da pesquisa, identificados como pontos fortes, mostram que, na percepção dos alunos e egressos participantes da pesquisa, o maior ponto forte da EGP são os docentes qualificados para a atividade de ministrar aula pelo fato de deter domínio dos conteúdos ministrados, isso indica que os critérios de seleção de docentes adotados pela EGP são eficientes para atender de forma qualificada a demanda pelos cursos. Na sequência aparece o fato de que há compromisso da direção da EGP com a formação do aluno, a preocupação dos professores e coordenadores para que a ementa e conteúdo de cursos sejam ministrados na íntegra, a importância dos aspectos relacionados a adequada matriz do curso e recursos pedagógicos, todos esses itens estão com mais de $90,0 \%$ de concordância dos entrevistados. No entanto, há baixa frequência no fato da biblioteca não ter referências suficientes e atualizadas, indicando a necessidade de adequação das ementas ao rol de livros, periódicos e outros produtos a disposição na biblioteca. $O$ item com a menor porcentagem que o identifique como um ponto forte foi o relacionado a precariedade dos laboratórios de informática.

Os resultados mostram que a grande maioria dos itens identificados como pontos fortes apresentam alto nível de qualificação na escolha, o que reforça a escolha feita pelo entrevistado em relação ao ponto forte. Há evidências de uma correlação negativa entre a porcentagem de identificação de ponto forte e a sua qualificação, indicando que os itens com baixo poder de identificação como ponto forte estão associados às porcentagens elevadas dos níveis Altos e Baixos de qualificação das respostas. Nesse contexto, os orientadores do PEI da EGP devem considerar tais aspectos quando da definição das ações internas de concertação das atividades desenvolvidas pela EGP. Esses resultados vêm ao encontro dos resultados encontrados com a sistematização das barreiras identificadas pela pesquisa junto aos servidores públicos.

Por fim, foi inquirido a avaliar o curso em relação a outros disponíveis no mercado amplo e não o mercado institucional composto pelas escolas de governo das três esferas administrativas e o resultado o seguinte: dos 80 entrevistados $37,5 \%$ afirmaram que o curso foi melhor, $31,5 \%$ disseram ser igual, $16,3 \%$ ser menos competitivo, $11,3 \%$ mais competitivo, e apenas $2,5 \%$ afirmaram ser pior do que o oferecido no mercado. Esses números mostram, em grande medida, o quanto os entrevistados estavam satisfeitos com o curso realizado na EGP.

Quando solicitado ao entrevistado (Aluno e Egresso) identificar quais itens ele entendia como oportunidades para que as suas habilidades e competências pudessem ser utilizadas em prol da melhoria do serviço público e por isso ele se dispôs a participar dos serviços ofertados pela EGP 


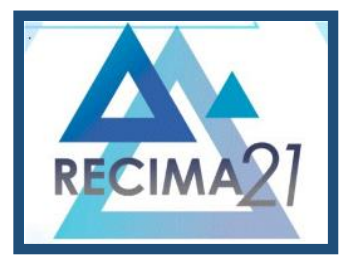

\section{RECIMA21 - REVISTA CIENTÍFICA MULTIDISCIPLINAR}

IDENTIFICAÇÃO E CARACTERÍSTICAS DOS STAKEHOLDERS NA CONSTRUÇÃO DO PLANEJAMENTO

ESTRATÉGICO DE UMA INSTITUIČ̃̃O PÚBLICA

Sérgio Castro Gomes, Laurimar De Matos Farias, Luciana Rodrigues Ferreira, Flavio Heleno Solano Reis

(cursos rápidos, pós-graduação, palestras, seminários, workshop), quase a totalidade deles afirmou que a participação nos cursos cria ou criaram oportunidades para que ele melhorasse a qualidade das atividades funcionais buscando a eficácia/efetividade.

O processo de Regionalização da Gestão Pública Estadual é visto como uma oportunidade pelo fato da descentralização da gestão administrativa do Estado ser algo necessário para que os serviços públicos sejam alocados de forma mais eficiente e os mecanismos de monitoramento e avaliação dos programas implantados pelas secretarias conforme definido nos Planos Plurianuais (PPA) se tornem efetivos.

Após a distribuição das oportunidades identificadas pelos Alunos e Egressos, de acordo com a frequência de ocorrência, foi avaliado o grau de qualificação do item, em que a oportunidade com maior proporção foi classificada como sendo de Alto ou Médio nível representando 99,0\%. Esse resultado mostra a elevada aderência entre o item identificado como oportunidade e a qualificação dele e que os entrevistados veem que podem melhorar a qualidade das suas atividades desde que sejam estimulados pelos gestores públicos.

A oportunidade relacionada ao processo de regionalização da gestão administrativa apresenta forte aderência entre a identificação e a qualificação desse item mostrando que os entrevistados enxergam na descentralização oportunidades de novas experiências e para isso buscaram as atividades proporcionadas pela EGP para que elevassem o nível de domínio das competências pessoais e de suas habilidades.

As oportunidades relacionadas a política de socioeconomia para o Estado também é indicada pelos entrevistados como oportunidade, porém, $12,0 \%$ e $23,0 \%$, respectivamente classificam elas como de baixa qualificação, com efeito tem-se a redução da aderência entre a identificação e a qualificação, o que pode ser explicado, em parte, pelo fato da gestão pública ainda não se estruturar para que a política de socioeconomia se torne realidade.

\subsubsection{Professores}

A amostra de professores que participaram da pesquisa mostrou que a média de idade deles foi de 45 anos, em que a menor idade foi de 26 anos e a maior 71 anos, em que o desvio padrão alcançou 11,2 anos. Aplicado o teste de normalidade Kolmogorov-Smirnov concluiu-se que a idade se distribuição conforme a curva normal; 50,0\% dos professores entrevistados apresentavam até 45 anos de idade; $50,0 \%$ dos entrevistados foram mulheres; $50,0 \%$ dos professores tinham curso de pós-graduação lato sensu e 47,0\% com titulação no Stricto Sensu (Mestrado e Doutorado); 60,0\% deles atuam como professores e $30,0 \%$ como técnicos no setor público.

Os dados sobre os pontos fortes percebidos pelos professores mostram o compromisso da EGP com formação profissional do servidor público. Há compromisso da direção e coordenação com a formação do aluno, currículos atualizados e planos de ensino adequados e Formação dos 


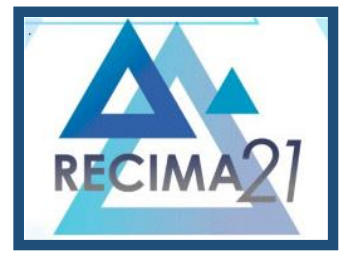

\section{RECIMA21 - REVISTA CIENTÍFICA MULTIDISCIPLINAR}

IDENTIFICAÇÃO E CARACTERÍSTICAS DOS STAKEHOLDERS NA CONSTRUÇÃO DO PLANEJAMENTO

ESTRATÉGICO DE UMA INSTITUIČ̃̃O PÚBLICA Sérgio Castro Gomes, Laurimar De Matos Farias, Luciana Rodrigues Ferreira, Flavio Heleno Solano Reis

servidores públicos adequada às necessidades do Estado, apresentaram acima de 90,0\% indicação como pontos fortes.

No entanto, os itens com as menores porcentagens foram Política de Relacionamento da EGP com os egressos, e Conhecimento da Missão e do Plano de Desenvolvimento da EGP, com $70,0 \%$ e 73,0\% respectivamente, isso mostra a necessidade da EGP formular e implementar uma política de comunicação e relacionamento com antigos professores e egressos como forma de avaliar suas atividades e corrigir os desvios das boas práticas que são percebidas pelos professores e servidores.

$\mathrm{Na}$ distribuição da qualificação dos itens indicados como pontos fortes pelos professores em que se observa aderência entre a identificação e a qualificação dos itens. Isso mostra que há certa homogeneidade de que a EGP tem compromisso com a formação do servidor público, e que a efetiva participação da direção geral e dos coordenadores das atividades é que a formação dos servidores públicos está adequada as necessidades do estado. Em relação aos itens com baixa aderência, em que não há convergência entre identificação e qualificação do item definido como ponto forte e isso mostra que no planejamento da EGP aspectos relacionados a política de comunicação e marketing institucional, atuação administrativa e financeira dos gestores, melhorar a divulgação da missão, visão e as estratégias da EGP devem ser intensamente divulgadas, e o ponto mais importante é a falta de um canal de relacionamento com o egresso da escola o que compromete o planejamento de ações futuras pois não se tem informação para saber em que medida a participação nos cursos de formação da EGP foi capaz de ampliar as habilidades, competências e capacidades dos servidores públicos para melhor desenvolver suas atividades.

No conjunto de oportunidades, os professores foram unânimes em afirmar que a formação de uma governança compartilhada para orientar a promoção do desenvolvimento sustentável do Estado é a grande oportunidade para EGP, pois a partir das ações da escola pode-se capacitar gestores e líderes que farão a gestão pública ancorada nos princípios da gestão por resultado e orientarão a elaboração e implementação de um planejamento estratégico que conduza a criação de valor público para os cidadãos.

O item de oportunidade com maior aderência entre a identificação e a qualificação é o relacionado ao desafio da formação de servidores para o desenvolvimento sustentável do Estado, essa convergência mostra o quanto os professores acreditam que a EGP tem papel fundamental nesse processo de formação de servidores para promover as práticas de gestão ancoradas na tecnologia da informação e em processos inovativos que possibilitem desenvolver a gestão em todo o território do estado e fazer emergir um sistema de Escola de Governança Pública do Estado do Pará em que o estado seja capaz de estabelecer e coordenar as prioridades dos vários atores, atuando menos como interventor e mais como regulador e mediador. 


\section{RECIMA21 - REVISTA CIENTÍFICA MULTIDISCIPLINAR}

IDENTIFICAÇÃO E CARACTERÍSTICAS DOS STAKEHOLDERS NA CONSTRUÇÃO DO PLANEJAMENTO

ESTRATÉGICO DE UMA INSTITUIČ̃̃O PÚBLICA

Sérgio Castro Gomes, Laurimar De Matos Farias, Luciana Rodrigues Ferreira, Flavio Heleno Solano Reis

Os itens relacionados a implementação da Política estadual de Socioeconomia e dos Conselhos Regionais de Governo apresentam baixa aderência entre a identificação e a qualificação, o que mostra a falta de entendimento sobre essa política pública intencionada pelo estado para viabilizar a descentralização e a melhor alocação dos recursos públicos e a utilização dos recursos naturais.

\section{DISCUSSÕES}

A luz do proposto por Bryson (1988; 2004), as duas primeiras etapas do processo foram executadas a partir de reuniões da direção geral com todos os colaboradores internos e externos a EGP, nesses encontros foi apresentado plano de ação para executar o PEI da escola e definido o grupo de trabalho que coordenou as atividades com publicação do ato oficial no diário oficial do estado de maneira que se deu publicidade ao ato.

Em reunião prévia foi definida a nova missão da EGP que é "Propor e executar políticas públicas de formação e desenvolvimento de pessoas e produção de conhecimento para o fortalecimento da capacidade de Escola de Governança Pública do Estado do Pará, visando progresso social do Estado". E a visão "Ser referência em Escola de Governança Pública do Estado do Pará".

A definição dos stakeholders do processo de planejamento incluiu os egressos e professores da EGP ampliando o conjunto de grupos de interesse do planejamento Bryson (2004). A inclusão desses grupos contribuiu para elevar a quantidade e a qualidade da informação produzida e que orientará as etapas seguintes do PEI. A identificação adequada desses grupos possibilita maior troca de informações sobre fluxos de processos e produtos, assim como a produção de conhecimento Bryson; Edwards; Van Slyke (2018). Esse fluxo de informações proporciona a instituição o amadurecimento de suas ações e orienta a formulação do PEl.

$\mathrm{Na}$ etapa de identificação das forças e fraquezas foram feitos dois levantamentos, em que os resultados se complementam, uma vez que foram dois grupos distintos os servidores da EGP e seus colaboradores, e os alunos, egressos e professores que são atores importantes para que as informações dos últimos sejam analisadas pelos primeiros. Na percepção dos funcionários os principais pontos fracos referem-se a estrutura física, falta de sistema informatizado para fazer a gestão dos cursos, falta da estratégia de comunicação e divulgação, assim como a avaliação dos egressos. Enquanto, os alunos, egressos e professores reforçam as indicações sinalizando que os laboratórios de informática devem ser mais bem estruturados, o acesso ao acervo da biblioteca deve ser ampliado, a necessidade de uma biblioteca virtual para atender aos cursos à distância. Com relação aos pontos fortes os que mais se destacam são: a credibilidade da EGP, a qualidade de seus cursos e professores e o quadro de servidores técnicos. Porém, fica a desejar a atuação administrativa e financeira da escola e a ausência de relacionamento com o egresso. 


\section{RECIMA21 - REVISTA CIENTÍFICA MULTIDISCIPLINAR}

IDENTIFICAÇÃO E CARACTERÍSTICAS DOS STAKEHOLDERS NA CONSTRUÇÃO DO PLANEJAMENTO

ESTRATÉGICO DE UMA INSTITUIČ̃̃O PÚBLICA

Sérgio Castro Gomes, Laurimar De Matos Farias, Luciana Rodrigues Ferreira, Flavio Heleno Solano Reis

As principais ameaças identificadas pelos servidores e colaboradores externos foram a preocupação com os fatores subjacentes a redução da demanda de servidores qualificados pela escola, o que pode ser comprovado pelos números de registros do núcleo de planejamento para o ano de 2012 com 23.957 servidores, e em 2017, esse número reduz para 14.678. Um dos fatores de explicação dessa redução é apontado pelos funcionários como sendo a falta de planejamento dos órgãos na programação de uso dos recursos de programas de formação de habilidades e competências previstas no Plano Plurianual (PPA) desses órgãos.

No que se refere às oportunidades, a ampliação da oferta de cursos na modalidade a distância é o consenso entre os grupos pesquisados, no entanto, esse é o maior desafio da EGP, pois requer a definição e implantação de uma política de ensino a distância para escola. Com efeito, tem-se a possibilidade de ampliar o número de servidores participando de cursos de pós-graduação stricto sensu e o nível das competências individuais e coletivas, entendendo-se que a competência é uma combinação de habilidades, de natureza institucional, coletiva e individual, que promovem o conhecimento e orientam as atitudes - tomada de decisão - com o objetivo de criar valor às organizações, no sentido do direcionamento das ações destas partes em busca do alcance dos objetivos organizacionais como enunciados por Quin (2003), Ruas (2001) e Teixeira (2012).

Diante dessas informações produzidas e sistematizadas pelas pesquisas, os coordenadores do PEI da EGP contam com um insumo valioso para enunciar os objetivos estratégicos e definir as ações a serem efetivadas como forma de garantir o atingimento da missão. Neste processo, a densidade de informação produzida a partir da percepção dos stakeholders faz a diferença para que o PEI da EGP possa ter o reconhecimento de cada um dos grupos que participou de forma tal que as ações venham atender as demandas desses grupos.

\section{CONCLUSÃO}

Os stakeholders primários da EGP identificados na pesquisa foram prestadores de serviços de segurança, higienização e Tecnologia da Informação (TI); Secretários, Diretores e Presidentes de organizações públicas; alunos; professores e egressos.

O estudo demonstrou que superar as lacunas de melhor estrutura dos laboratórios de informática, o acervo da biblioteca e a logística tecnológica para a efetivação do ensino à distância são necessárias para que se atenda, de modo efetivo, o que foi apontado na percepção dos stakeholders.

Superar as barreiras internas é uma tarefa que se apresenta necessária para que as relações intra e interinstitucional possam se desenvolver e garantir o fluxo cotidiano da função do órgão junto ao estado. Estas prerrogativas estabeleceram o entendimento e, consequentemente, 0 interesse dos gestores da EGP pela formulação, a partir dos princípios basilares, do planejamento estratégico da instituição, levando em consideração um modelo de planejamento que acolhe uma 


\section{RECIMA21 - REVISTA CIENTÍFICA MULTIDISCIPLINAR}

IDENTIFICAÇÃO E CARACTERÍSTICAS DOS STAKEHOLDERS NA CONSTRUÇÃO DO PLANEJAMENTO ESTRATÉGICO DE UMA INSTITUIČ̃̃O PÚBLICA Sérgio Castro Gomes, Laurimar De Matos Farias, Luciana Rodrigues Ferreira, Flavio Heleno Solano Reis

gestão participativa onde as percepções e contribuições de um grupo de stakeholders foram levadas em consideração, permitindo, assim, um planejamento mais horizontalizado, isto é, as proposições e a operacionalização do plano da EGP têm a participação de todos aqueles que formam o coletivo institucional, portanto, são responsáveis pela garantia de cumprimento, a médio e longo prazo, da consecução do que foi proposto, conforme pode-se verificar na análise até aqui desenvolvida.

\section{REFERÊNCIAS}

AIRES, Renan Felinto de Farias et al. Escolas de Governo: o panorama brasileiro. Rev. Adm. Pública, Rio de Janeiro, v. 48, n. 4, p. 1007-1027, Aug. 2014. Disponível em: http://www.scielo.br/scielo.php?script=sci arttext\&pid=S0034-76122014000400010\&lng=en\&nrm=iso. Acesso em: 9 jul. 2019.

BATISTA, Fábio Ferreira. Modelo de gestão do conhecimento para a administração pública brasileira: como implementar a gestão do conhecimento para produzir resultados em benefício do cidadão. Brasília: Ipea, 2012.

BIDERMAN, Ciro; ARVATE, Paulo Roberto. Economia do setor público no Brasil. Rio de Janeiro: Elsevier, 2004.

BOURNE, Lynda; WALKER, Derek H. T. Visualising and mapping stakeholder influence. Management decision, v. 43, n. 5, p. 649-660, 2005.

BRYSON, J. M. What to do when Stakeholders matter. Public Management Review, v. 6, n. 1, p. 2153, 2004.

BRYSON, J. M.; ROERING, W. D. Initiation of Strategic Planning by Governments. Public Administration Review, v. 48, n. 6, p. 995-100. nov/dec. 1988.

BRYSON, John M.; EDWARDS, Lauren Hamilton; VAN SLYKE, David M. Getting strategic about strategic planning research. Public Management Review, v. 20, n. 3, p. 317-339, 2018.

CRESWELL, J. W. Projeto de pesquisa: métodos qualitativo, quantitativo e misto. 3. ed. Porto Alegre: Artmed, 2010.

FREEMAN, Edward R. Stakeholder theory - the state of the art. Cambridge: Cambridge University Press, 1984.

KAPLAN, Robert S.; NORTON, David P. A estratégia em ação: balanced scorecard. Rio de Janeiro: Campus, 1997.

NOGUEIRA. Marco Aurélio. Um Estado para a sociedade civil: temas éticos e políticos da gestão democrática. São Paulo: Cortez, 2005. p.37 a 54.

PACHECO, R. S. Escolas de Governo como centros de excelência em gestão pública: a perspectiva da Enap - Brasil. Enap Revista do Serviço Público, Brasília, v. 53, n. 1, jan/mar. 2002.

Escolas de governo: tendências e desafios - Enap-Brasil em perspectiva comparada. Enap Revista do Serviço Público, Brasília, v. 51, n. 2, p. 35-53, 2000.

PHILLIPS, R. A., FREEMAN, R. E.; WICKS, A. C. What stakeholder theory is not. Business Ethics Quarterly, v. 13, p. 479-502, 2003. 


\section{RECIMA21 - REVISTA CIENTÍFICA MULTIDISCIPLINAR}

IDENTIFICAÇÃO E CARACTERÍSTICAS DOS STAKEHOLDERS NA CONSTRUÇÃO DO PLANEJAMENTO

ESTRATÉGICO DE UMA INSTITUIÇÃO PÚBLICA Sérgio Castro Gomes, Laurimar De Matos Farias, Luciana Rodrigues Ferreira, Flavio Heleno Solano Reis

QUIN, Robert E. et al. Competências gerenciais: princípios e aplicações. Tradução de Cristiana de Assis Serra. Rio de Janeiro: Elsevier, 2003.

RUAS, R. Desenvolvimento de competências gerenciais e contribuição da aprendizagem organizacional. In FLEURY, M.T.L. e OLIVEIRA JR. M.M. Gestão estratégica do conhecimento: integrando aprendizagem, conhecimento e competências. São Paulo: Atlas, 2001.

SAVAGE, G.; NIX, T.; WHITEHEAD, C.; Blair, J. Strategies for assessing and managing organizational stakeholders. Academy of Management Executive, v. 5, n. 1, p. 61-75, 1991.

TEIXEIRA, Vilmar Agapito. Criando Valor Público - Gestão Estratégica no TCU. Brasília, 2012. Disponível em: http://portal2.tcu.gov.br/portal/page/portal/TCU/educacao corporativa/lideranca. Acesso em: 02 jul. 2019. 